\section{Endo-urologie en stenen}

\author{
Robert J.A. Oomen - A.C. (Carolien) Bouma-Houwert
}

\section{Introductie}

Hoewel het congres in Amsterdam dit jaar geen doorgang heeft gevonden, is er toch een hoop te vertellen over de urologie. Dat blijkt uit het uitgebreide programma dat geheel virtueel werd gepresenteerd dit jaar. 'Hot Shots' uit alle vakgebieden schoven aan om iedereen bij te praten over hun passie. Het merendeel van de praatjes betrof oncologische onderwerpen, maar er is zeker ook het een en ander te melden op het gebied van stenen en endo-urologie

\section{Voorspellen}

Niet alleen in de oncologie doen nomogrammen het goed. Er zijn meerdere onderzoeken naar voorspellende factoren bij urolithiasis. In de MIMIC-studie (Multi-centre Cohort Study Evaluating the role of Inflammatory Markers in Patient's Presenting with Acute Ureteric Colic), door een Britse groep jonge urologen (BUST-groep), werden in een multicenterstudie met een cohort van ruim 4.000 patiënten, factoren geëxtraheerd die spontane steenpassage kunnen voorspellen [1]. De belangrijkste parameters zijn steengrootte, positie en neutrofiele granulocyten. Het ontwikkelde nomogram is intern en extern gevalideerd en de groep is een app aan het ontwikkelen. Bij een steenbehandeling kan het handig zijn om iets van de samenstelling van de steen te weten. Met Dual-Energy CT is goed onderscheid te maken tussen vier veelvoorkomende steentypen [2]. Ook is op basis van CT goed te voorspellen of de steen geïmpacteerd is, met name door hydronefrose, maar ook door de HU-waarden boven en onder de steen te meten [3]. Door een 3D-reconstructie van de CT te maken, kun je in virtual reality door de nier navigeren. Dit is te gebruiken om patiënten uitleg te geven [4], maar kan ook gebruikt worden om een goede berekening van de infundibulopelvische hoek te maken, anders dan met de eerder beschreven methoden [5]. Met (gratis!) 3D-software is het mogelijk om beelden uit PACS te importeren, en te berekenen hoe lang de lasertijd is per type laser en per instelling van de laser [6].

drs. R. J. A. Oomen $(\triangle)$ · drs. A. C. Bouma-Houwert afdeling Urologie, St. Antonius Ziekenhuis Nieuwegein, Nederland r.j.a.oomen@gmail.com
Als we dan overgaan tot behandelen, is het handig om te weten of de geplande ureterorenoscopie (URS) makkelijk of moeilijk uitvoerbaar is. Het ScorDiS-nomogram helpt hierbij en is gebaseerd op de karakteristieken van 216 ingrepen door een ervaren endo-uroloog [7]. In een grote Britse database $(n=4004)$ zijn factoren gevonden die postoperatieve infectie na URS voorspellen. De odds ratio's (OR's) voor infectie zijn voor het vrouwelijk geslacht en multipele stenen ongeveer 2 [8].

Ook de uitkomst van extracorporal shockwave lithotripsy (ESWL) is te voorspellen. Een Amerikaans cohort werd geanalyseerd en met deep learning algoritmes werd een nomogram opgesteld [9]. Helaas houden de Amerikanen de belangrijke predictoren voor zichzelf. Een calculator is wel in de maak.

De uitkomsten van percutane nefrolitholapaxie (PNL), zowel de stone free rate (SFR) als de complicaties, zijn met meerdere scoringssystemen te bepalen (CROES, S.T.O.N.E., GSS en ReSC). In een prospectieve analyse $(n=162)$ scoorden alle systemen goed. CROES voorspelde de SFR het beste en S.T.O.N.E. deed dit het beste voor complicaties [10]. Twee retrospectieve studies lieten ook zien dat de systemen elkaar niet veel ontlopen $[11,12]$.

\section{Nieuwe technieken, tips en tricks}

Vorig jaar waren al de eerste preklinische en kleine klinische studies gedaan naar de Super pulsed thulium fiber laser (SPTFL) [13], maar ook afgelopen jaar heeft men niet stilgezeten. Oliver Traxer gaf in zijn webinar een uitstekende uitleg over de techniek ervan. In de pro-and-consessies wist Peter Kronenberg zijn tegenstander te verpulveren met een prachtige presentatie over alle voordelen van thulium boven holmium; een samenvatting staat in het kader.

In vitro geeft de SPTFL in kortere tijd meer en betere fragmentatie [14]. Uiteraard kan de SPTFL ook gebruikt worden voor PNL. De ervaringen met de eerste 120 patiënten zijn goed [15]. Ook de eerste en-blockresectie van een blaastumor en incisies van een UPJ-stenose $(n=48$, recidief $4,2 \%$ ) zijn al een feit met de SPTFL $[16,17]$. Holmium is zeker niet afgeschreven; met pulsmodulatie is het een erg krachtige en effectieve laser (Holmium 2.0 presentatie door Ghani). 


\section{Voordelen van SPTFL boven Ho:YAG [13]}

- Fragmentatie twee keer sneller

- Dusting vier keer sneller

- Meer partikels $<0,1 \mathrm{~mm}$

- Minder retropulsie

- Kleinere laserfibers (tot $50 \mu \mathrm{m}$ )

- Lage energiesettings (tot 0,025 J)

- Hoge frequentie (tot $2400 \mathrm{~Hz}$ )

- Langere pulsduur (tot $50 \mathrm{~ms}$ )

- Gewicht van de machine (35 kg vs. $300 \mathrm{~kg}$ )

- Kleiner formaat, past in endoscopietoren

- Minder energieconsumptie (800 vs. $10.000 \mathrm{~W}$ )

- Geen krachtstroom op OK nodig

- Minder lawaai (geen waterkoeling)

Ook zeer interessant is de EMS Lithoclast Trilogy. Pneumatisch en ultrasoon gedreven en met behulp van suctie zorgt dit apparaat voor een veel snellere steenbehandeling dan conventionele dual energy lithotrypsieapparaten. Wel ging 9\% van de probes kapot. De oorzaak is nog onduidelijk (productie, verkeerd gebruik, etc.) [18].

In een meta-analyse van vier randomized controlled trials $(n=284)$ liet men zien dat het lokaal infiltreren met anesthetica rondom het PNL-traject minder postoperatieve pijnklachten geeft bij een 'tubeless' PNL [18]. Het inspecteren en zo nodig nacoaguleren van het traject na PNL gaf in een vergelijkende studie $(n=485)$ minder postoperatieve bloedingen in de coagulatiegroep [20].

Een aanrader zijn de ESU-courses die allemaal gemakkelijk terug te vinden zijn op YouTube (zoek op 'urowebinar' of ga naar het kanaal van de 'European School of Urology').

\section{Veiligheid}

In een aantal sessies werd ook ingegaan op veiligheidsaspecten. Met alle nieuwe lasers willen we steeds sneller gaan, maar kan dat zomaar? Bij een te hoge druk, dit is een intrapelvische druk > 30-40 mmHg, treedt terugstroom op en is de kans op complicaties, zoals sepsis groter (Plenaire sessie 6: Liatsikos). Het gebruik van een access sheet verlaagt de druk aanzienlijk. Ook het gebruik van een irrigatiepomp en prestenting zorgen ervoor dat er veel minder hoge drukken in de nier ontstaan.

Temperatuurverhoging werd beschreven in een prachtig mathematisch model [21]. De onderzoeksgroep concludeert dat het gebruik van verwarmde vloeistoffen bij laserlithotrypsie de temperatuur vaak boven de ('kritische') $43^{\circ} \mathrm{C}$ opdrijft. Ook vermindert een irrigatiepomp de maximale temperatuur drastisch [22]. De temperatuurverhogingen tussen thulium en holmium zijn vergelijkbaar [23].

Een retrospectieve studie bij 114 patiënten liet zien dat een URS tijdens opname voor pyelonefritis net zo effectief en veilig is als een uitgestelde ingreep [24]. Complicaties werden echter niet op langere termijn bijgehouden.

\section{Kosten}

Steenlijden kost de maatschappij veel geld. De geschatte kosten in het Verenigd Koninkrijk zijn vergelijkbaar met de jaarlijkse kosten voor blaas- en prostaatkankerzorg samen (ca. $£ 235$ miljoen) [25]. In 2017 werd door Martin et al. beschreven dat in een laagvolumecentrum $(<100$ per jaar) de wegwerp-ureterorenoscoop kosteneffectief is [26]. Onze Belgische collega's deden ook onderzoek naar de kosteneffectiviteit [27]. Afhankelijk van het merk wegwerpscoop is een herbruikbare scoop na 155-274 casus goedkoper. Een procedure met een herbruikbare flexibele scoop kost vanaf de $400^{\mathrm{e}}$ ingreep ongeveer $€ 480$.

\section{Take home message}

- We gaan steeds meer naar een patiëntgebonden aanpak, waarbij we gebruik kunnen maken van rekenmodellen en nomogrammen om de optimale behandeling voor de patiënt te waarborgen.

- De SPTFL is een veilige en veelbelovende laser; meer studies en toepassingen zullen volgen.

- Druk- en temperatuurveranderingen kunnen schadelijk zijn. Ureterorenoscopen zullen in de toekomst uitgerust worden met druk- en temperatuursensoren zodat steeds veiliger gewerkt kan worden.

- Ook het milieu en kosten gaan waarschijnlijk een grotere rol spelen in de toekomst.

Open Access This article is distributed under the terms of the Creative Commons Attribution 4.0 International License (http:// creativecommons.org/licenses/by/4.0/), which permits unrestricted use, distribution, and reproduction in any medium, provided you give appropriate credit to the original author(s) and the source, provide a link to the Creative Commons license, and indicate if changes were made.

\section{Bronnen}

1. Gao C, Peters M, Jayaraajan K, et al. Development of a risk calculator to predict spontaneous stone passage in patients with acute ureteric colic. EAU20 Virtual, abstract, poster, presentation 789 .

2. Nazarov T, Rychkov I.V, Trubnikova K, et al. The clinical using of dual-energy computed tomography and its potential in determining the biomineralogical composition of stones. EAU20 Virtual, PT107.

3. Deguchi R, Yamashita S, Kikkawa K, Kohjimoto Y, Hara I. HU above-below ratio is an useful preoperative factor for predicting impacted ureteral calculi. EAU20 Virtual, abstract, poster, presentation 542 .

4. Guliev B, Komyakov B, Talyshinskii A 'InsKid' - the app displaying inside view of 3D-reconstructed PCS. Is it clearer for patients? EAU20 Virtual, abstract, video.

5. Marra G, Zhuang J, Beltrami M, et al. The mobile app displaying inside view of the kidney's PCS and measuring infundibulopelvic angle. Is there any difference with the existing methods? EAU20 Virtual, abstact V008.

6. Panthier F, Doizi S, Berthe L, Traxer O, Groupe de Recherche Clinique sur la lithiase urinaire $\left(\mathrm{n}^{\circ} 20\right) 4$. Developing a free three-dimensional tool for kidney stones' surgical planning: 'CALCULATOR'. EAU20 Virtual, abstract, poster, presentation 544. 
7. Dal Moro F, Borso C, De Giorgi G, et al. How to identify an 'easy' retrograde intra-renal surgery to be performed by naïve residents in defining a training program? ScorDiS Nomogram. EAU20 Virtual, abstract, poster, presentation 554.

8. Campbell of Dunstaffnage AAE, Birks J, Turney BW. Predicting sepsis after ureteroscopy: Outcome analysis of 4768 patients from a national data registry in the United Kingdom. EAU20 Virtual, abstract, poster, presentation 553.

9. Seltzer RGN, Hamilton BD, Klett DE, Chen Z, Nakada SY, Gerber GS. The prediction of treatment success and complications of shockwave lithotripsy using artificial intelligence. EAU20 Virtual, abstract, poster 119.

10. Elkaramany T, Al Adl A, Mohey A, Abedel Aal A, Abu Elnasr $\mathrm{H}$, Noureldin $\mathrm{Y}$. The first prospective assessment of S.T.O.N.E, Guy, CROES, S-ReSC nomograms in the prediction of percutaneous nephrolithotomy outcomes. EAU20 Virtual, abstract, poster, presentation 666 .

11. Bibi M, Ouanes Y, Zakhama W, Manitta M, et al. Do the scoring systems predict the success of percutaneous nephrolithotomie for staghorn stones? Comparison of 4 scores: The Guy's stone score, STONE Score, CROES nomogram and S-ReSC score. EAU20 Virtual, abstract, poster, presentation 667.

12. Lai S, Jiao B, Liu J, et al. Comparing different kidney stone scoring systems for predicting percutaneous nephrolithotomy outcomes: A multicenter study. EAU20 Virtual, abstract, poster, presentation 668 .

13. Traxer O, Keller EX. Thulium fiber laser: the new player for kidney stone treatment? A comparison with Holmium:YAG laser. World J Urol. 2020;38(8):1883-1894.

14. Panthier F, Doizi S, Berthe L, Traxer O, Groupe de Recherche Clinique sur la lithiase urinaire $\left(n^{\circ} 20\right) 3$. In vitro comparison of ablation rates between superpulsed thulium fiber laser and HO:YaG laser for endocorporeal lithotripsy. EAU20 Virtual, abstract, poster 131 .

15. Enikeev D, Taratkin M, Alyaev Y, et al. Super-pulse thuliumfiber laser for lithotripsy: First clinical experience in percutaneous nephrolithotomy. EAU20 Virtual, abstract, poster, presentation 181.

16. Enikeev D, Taratkin M, Laukhtina E, et al. Safety and shortterm oncological outcomes of thulium fiber laser en-bloc resection of non-muscle-invasive bladder cancer: A prospective non-randomized phase II trial. EAU20 Virtual, abstract, videopresentation 057.

17. Martov AG, Ergakov DV, Golubev MYu, Golubev PM, Baykov NA, Abdullaev DAl. Our experience at retrograde thulium fiber laser endopyelotomy. EAU20 Virtual, abstract, poster, presentation 439.
18. Wiseman O, Tanse F, Saeb-Parsy K, et al. The efficacy and safety of the EMS Lithoclast Trilogy: A European multicenter prospective study on behalf of ESUT. EAU20 Virtual, abstract, videopresentation 057.

19. Dechao F, Yunjin B, Yin T, Wuran W, Ping H. The efficacy of local anesthetic infiltration around nephrostomy tract on postoperative pain control after tubeless percutaneous nephrolithotomy: A meta-analysis of randomised control trials. EAU20 Virtual, abstract, poster 112.

20. Wang XL. Improving the safety of tubeless percutaneous nephrolithotomy by plasmakinetic hemostasis. EAU20 Virtual, 674 .

21. Goldsmith L, Williams JG, Moulton DE, Waters SL, Turney BW. A mathematical model of renal temperatures during laser lithotripsy. EAU20 Virtual, abstract, poster 128.

22. Kallidonis P, Noureldin Y, Adamou C, Ntasiotis P, Liatsikos E. Does forced irrigation buffer heat generated from highpower laser activation during retrograde intrarenal surgery? Experimental study in a live-anesthetized pig. EAU20 Virtual, abstract, poster, presentation 172.

23. Taratkin M, Laukhtina E, Singla N, Rapoport L, Enikeev D. Temperature changes during laser lithotripsy with Ho:YAG laser and novel Tm-fiber lasers: a comparative in-vitro study. EAU20 Virtual, abstract, poster, presentation 179.

24. Zaghbib S, Ouanes Y, Bibi M, et al. Safety and efficacy of early ureteroscopy after obstructive pyelonephritis. EAU20 Virtual, 549 .

25. Geraghty R, Cook P, Walker V, Somani BK. Evaluation of the economic burden of kidney stone disease in the UK: a retrospective cohort study. EAU20 Virtual, abstract, poster, presentation 781.

26. Martin CJ, McAdams SB, Abdul-Muhsin H, et al. The Economic Implications of a Reusable Flexible Digital Ureteroscope: A Cost-Benefit Analysis. J Urol. 2017;197(3 Pt 1):730735

27. van Compernolle D, Veys R, Beysens M, van Haute C, Tailly T. Cost-efficiency analysis of reusable versus single-use flexible ureteroscopy and the development of a formula to evolve to a cost-efficient hybrid model. EAU20 Virtual, abstract, poster, presentation 546.

drs. Robert J.A. Oomen aios urologie

drs. A. Carolien Bouma-Houwer uroloog, androloog 